\title{
A SIMPLE EQUATION OF STATE OF MATTER AT SUPER-NUCLEAR DENSITIES*
}

\author{
Y. C. LEUNG \\ Southeastern Massachusetts University, North Dartmouth, Mass. 02747, U.S.A. \\ and \\ C. G. WANG \\ Massachusetts Institute of Technology, Cambridge, Mass. 02139, U.S.A.
}

\begin{abstract}
The qualitative features of the equation of state of matter at supernuclear densities are deduced through a careful examination of the nature of particle interactions at short distances and by the introduction of an 'effective mass spectrum.' it is found that the equation of state begins to take on a particularly simple form (the 'asymptotic form') at a relatively low matter density of $10^{17} \mathrm{~g} \mathrm{~cm}^{-3}$. A brief review of various approaches to calculate the equation of state for a neutron star is also given.
\end{abstract}

\section{Introduction}

In a system of particles with strong interaction dynamics, the two models very often employed are the potential interaction and the dual resonance approach. The former has been applied very extensively, and most successfully, to systems of many nucleons; the latter has been applied to systems of a few (most successfully zero) baryons. In the construction of an equation of state for a neutron star, the matter at nuclear densities can be described very accurately by the potential model of two-neutron correlations, while at much higher densities, the proliferation of excited particle states suggests the dominance of single particle dynamics. Unfortunately, the region of density which is most sensitive to the mass of a neutron star is at a matter density of $10^{15}$ to $10^{17} \mathrm{~g} \mathrm{~cm}^{-3}$, and it is not covered by the usual potential interactions in nuclear physics, or by the dual resonance model in high energy physics. A major effort is clearly required to bring together techniques from different fields of physics to explain this wonderful object.

\section{Difficulties in the Theoretical Models}

Figure 1 shows some results of neutron matter calculations (Leung and Wang, 1971). The equations of state derived from various nuclear potentials are fairly consistent with each other for matter near nuclear densities, but they begin to diverge with each other at higher densities as the effect of the nuclear potential core becomes important. There are various forms of potential core; some are infinitely stiff at a finite core radius, some have an inverse radius dependence on the repulsion, some have finite and constant repulsion, and some have no explicit repulsion. It is not clear that one form of potential core is less arbitrary than the other. There are many corrections that should

* Presented by C. G. Wang. 


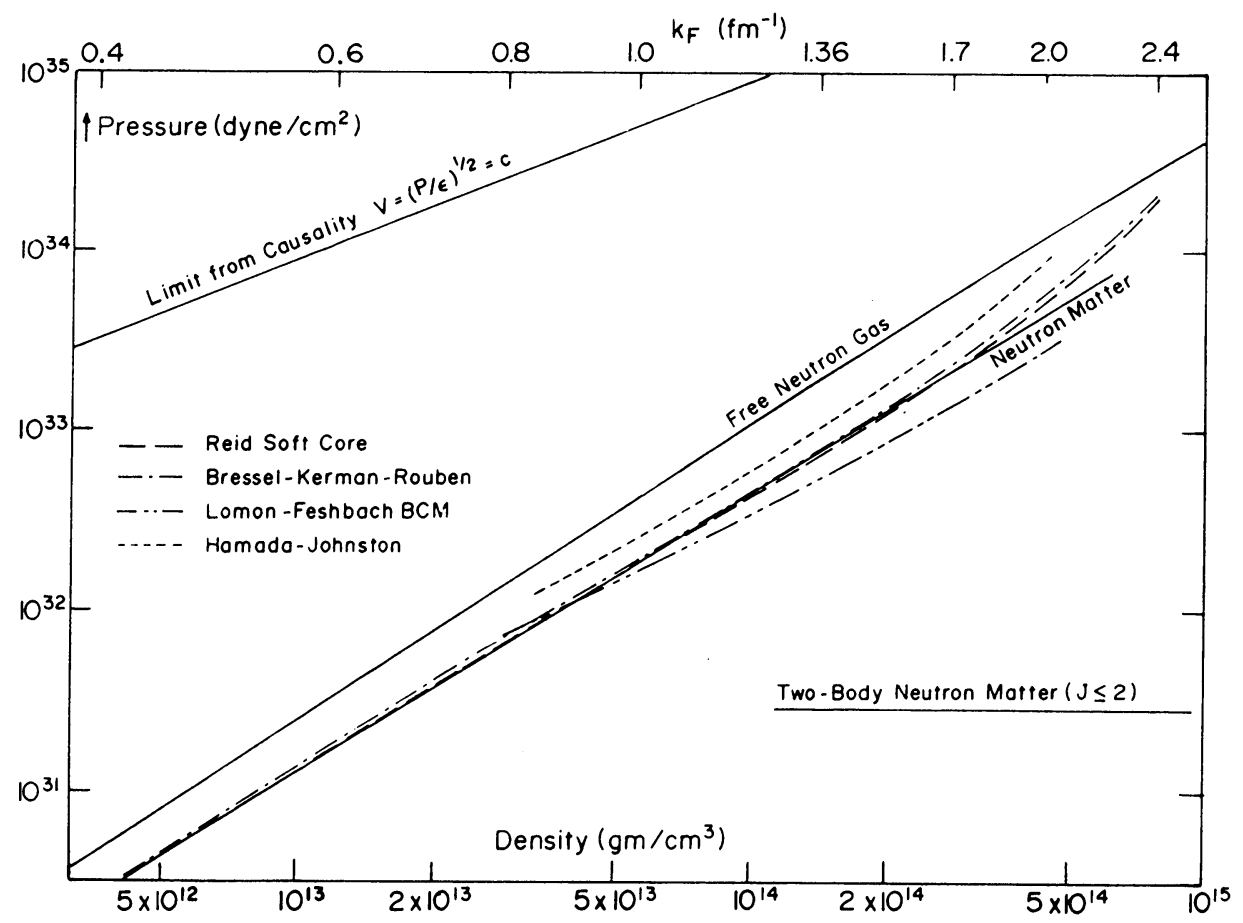

Fig. 1. Equations of state for neutron matter calculated from four sets of nuclear potentials.

be included in the consideration; the condensation energy of the neutrons, the clustering effect of the protons and the small contribution from the electrons, the many-body forces, the correlations from higher than the two-body terms, the relativistic corrections, the potential contribution due to the high angular momentum partial waves, the non-local consideration of the momentum transfer at short distances. Besides the first two items, all corrections become increasingly important as the nucleons get closer. There are also some basic questions that may be asked about the potential approach. Consider, for example, the Yang scaling model with $0.7 \mathrm{fm}$ for the proton dimension and $0.6 \mathrm{fm}$ for the pions. Surely when the internucleon distance approaches this dimension, some serious corrections should be made. The most important difficulty perhaps arises from the lack of a well developed potential to operate among hadrons in general. One may certainly consider all baryons as if they were nucleons, but there is no compelling reason to do so.

Figure 2 is an overall equation of state for degenerate matter. Starting with a very repulsive potential core and extending the many-body calculations from the nuclear physics, one obtains the maximum mass of a neutron star of about $1.5 M_{\odot}$, while without an explicit repulsion in the nuclear core, the limiting mass may be as low as $0.3 M_{\odot}$. Note that the equation of state is limited by the so-called 'causality relation' $P=\varepsilon c^{2}$; a stiff equation of state at matter densities higher than $10^{15} \mathrm{~g} \mathrm{~cm}^{-3}$ would not support a neutron star heavier than about $1.5 M_{\odot}$ (unless one let the equation of state 


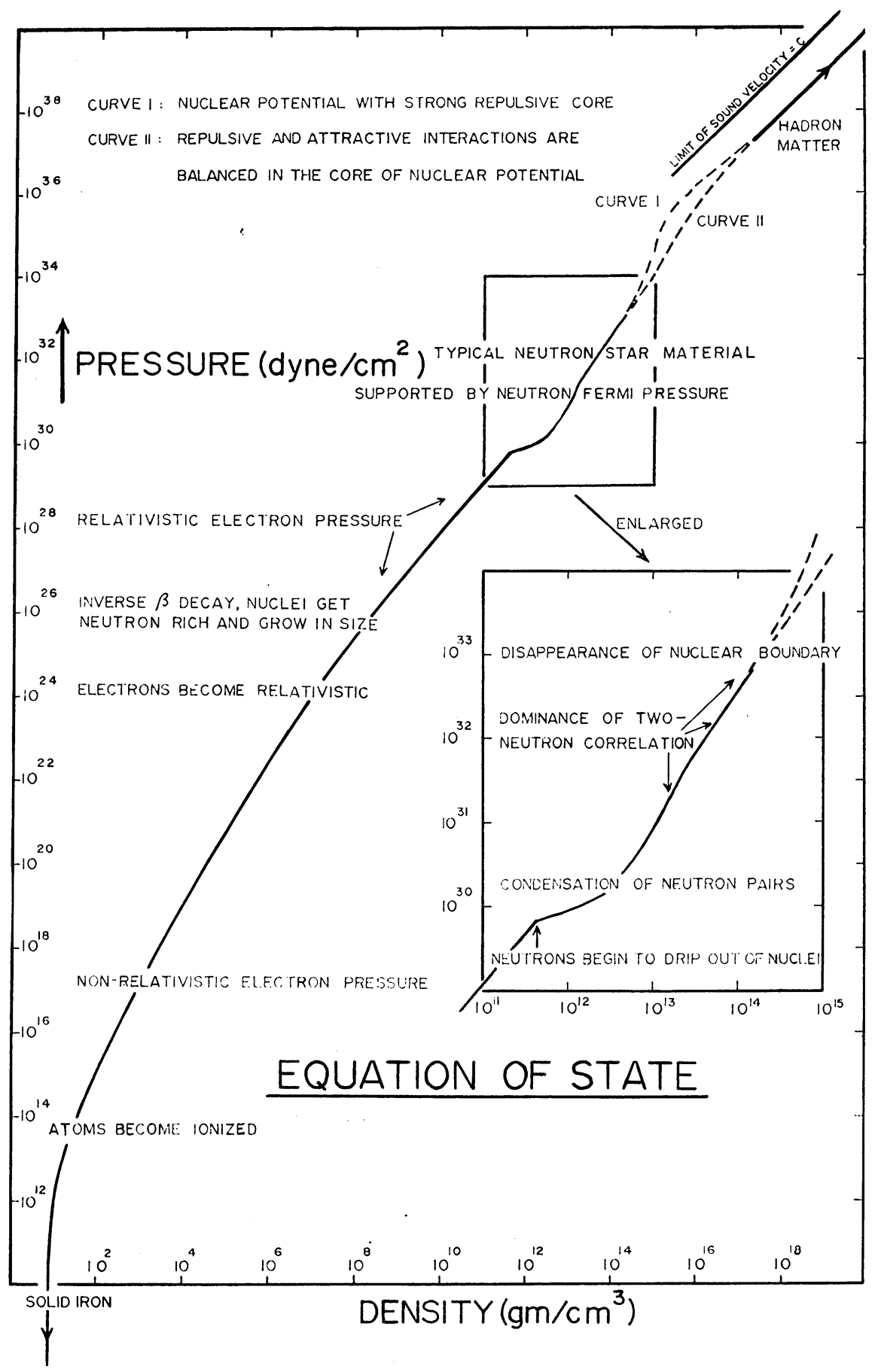

Fig. 2. An overall description of the equation of state for the cold degenerate matter maintained at neutral charge. Curve (1) can support a neutron star with maximum mass of about $1.5 M_{\odot}$, while curve (2) can support only $0.3 M_{\odot}$. 
go beyond the causality limit) - only a stiff equation of state at matter density around $10^{14} \mathrm{~g} \mathrm{~cm}^{-3}$ would do, but this is where the usual nuclear physics operates and a judgment can be easily made.

Raymond Sawyer in a recent series of articles (Sawyer, 1972a, 1972b), cast two serious doubts upon the recent calculations made on the equation of state of matter at supernuclear densities. His first objection is that the states of the decay channel of the excited baryons may be occupied and their self-energy should therefore be shifted upward. The second objection is that the pions in a very dense matter may form a degenerate sea so as to considerably reduce the Fermi energy of the baryons. These objections apply to the potential approach as well as to the dual resonance or the bootstrap considerations. We concur with Sawyer's observation, and suspect that some future considerations uncommon to the usual nuclear physics or high energy physics may yet to be raised. Faced with such extraordinary uncertainties, we come back to a re-examination of the baryons spectrum hoping to learn some general properties of the equation of state of matter at supernuclear densities.

\section{The Baryon Mass Spectrum}

A list of known baryons with masses below $2 \mathrm{GeV}$ can be found in an article by Söding et al. (1972). This list is fairly complete and is transcribed in Appendix A giving the mass and multiplicity of each. If we compare the cumulative number of baryons, $N$, below a certain mass with the square of that mass, $\mathrm{m}^{2}$, normalized to that of the neutron mass, $m_{\mathrm{N}}^{2}$, we can see from Figure 3 that it can be approximated by a simple power law:

$$
N=c_{1}\left(m^{2} / m_{\mathrm{N}}^{2}\right) a .
$$

In Figure $3, \log N$ is plotted against $\log \left(\mathrm{m}^{2} / \mathrm{m}_{\mathrm{N}}^{2}\right)$ and the data show remarkable linearity with $c_{1}=4.0$ and $a=2.9$. From (1) we can deduce the baryon spectrum:

$$
\Delta N(m)=c_{0}\left(m^{2} / m_{\mathrm{N}}^{2}\right)^{\alpha} \Delta\left(m^{2} / m_{\mathrm{N}}^{2}\right),
$$

where $\Delta N$ denotes the number of baryons within the mass interval $\Delta\left(m^{2}=m_{N}^{2}\right)$, and $c_{0}=a c_{1}, \alpha=a-1$.

Just as the free neutron equation of state served to illustrate the qualitative features of the equation of state of nuclear matter, the equation of state derived from free baryons by including all known baryons would provide a qualitative guide to that of supernuclear matter. Such an attempt was first considered by Ambartsumyan and Saakyan (1960) who made use of all hyperons and resonances known at that time with and without some form of interparticle interaction. Based on repulsive vector meson exchange, Zel'dovich (1961) has given

$$
P=(v-1) \mathscr{E} c^{2} \text {. }
$$

where $P$ is the pressure, $\mathscr{E}$ the energy density and $v=2$ in his asymptotic limit for repulsive interactions. However, Harrison (1965) claimed that due to multiple-meson 
exchange, the parameter $v$ would be brought back to the more conventional range, $1 \leqslant v \leqslant 4 / 3$.

The equation of state derived on the basis of free particles cannot of course be realistic and there are serious objections to it since baryons interact strongly at such interparticle distances. The neglect of strong interactions certainly cannot be justified. Two issues have been raised. First, we know that the mass of a particle resonance such as one of those listed in Appendix A is determined experimentally when the particle is free to decay strongly. However (Sawyer, 1972a), if the resonance is inhibited from decay due to the lack of phase space, as is the case when it is immersed in the Fermi sea of dense matter, would its mass remain the same? Second, strong interactions among baryons and among baryons and mesons (Sawyer, 1972b, Scalapino, 1972) can contribute substantial interaction energy to the system. How much is this interaction energy?

Since there is no accurate way of computing the mass shifts of resonances due to

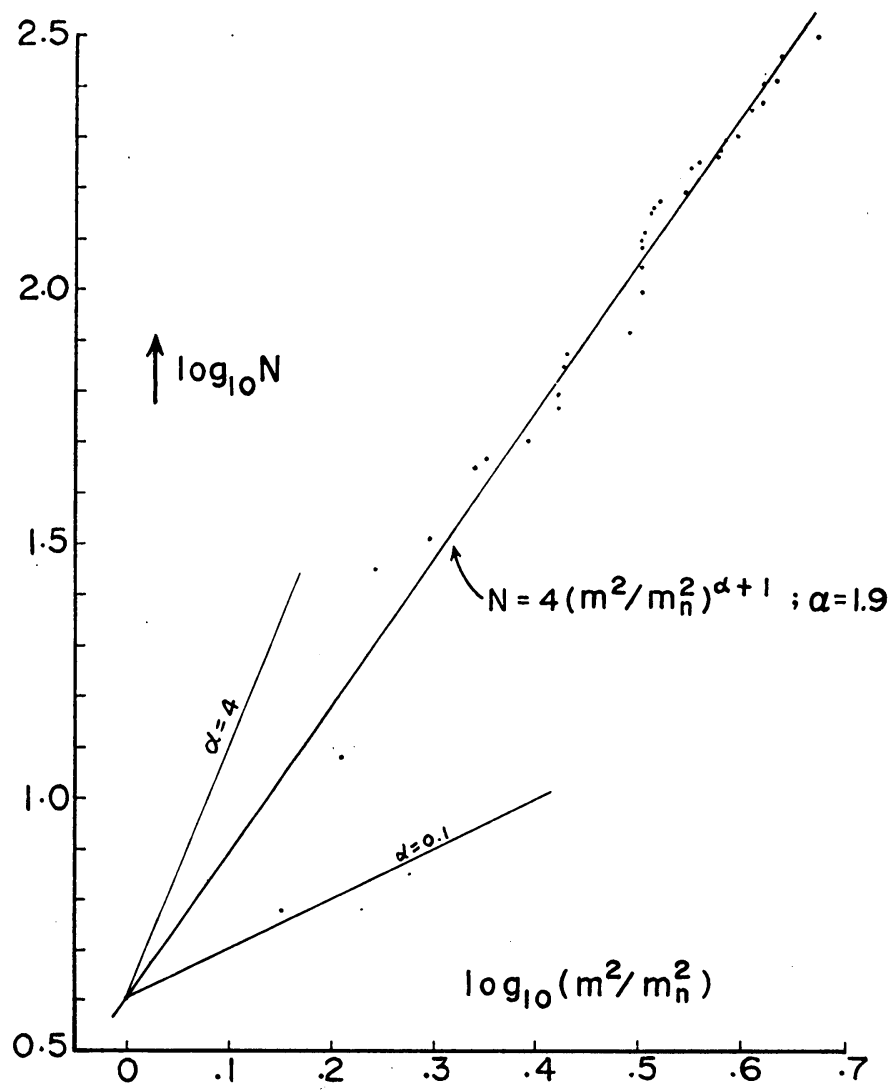

Fig. 3. The cumulative number of baryons $N$ below a certain mass $m$ is plotted against the square of the mass normalized with respect to the square of the nucleon mass $m_{N}$ on a $\log \log$ plot. The spectrum is very sensitive to the index $\alpha$. 
alterations in their lifetimes, and the knowledge of particle dynamics is at present inadequate to determine the interaction energy, the problem has reached an impasse. We shall nevertheless give some plausible arguments below which would then allow us to understand the qualitative nature of the equation of state at supernuclear densities. We shall make use of the notion of mass shifts and claim that the equation of state can be derived in terms of a free-particle formalism if the mass of each particle is shifted sufficiently to adjust for interaction. It is, of course, always possible to describe the statistical mechanics of an interacting baryon matter in terms of a free baryon gas provided the masses of particles are adjusted continuously to yield the correct result. This, however, is a very difficult thing to do in general if there are only a few types of baryons involved since the adjusted mass of each would have to be known exactly. Now, the main point of our argument is that since the baryon mass spectrum obeys a power law, it is most probable that the 'effective baryon mass spectrum' (which one would use in conjunction with a free-particle formalism) due to mass shifts as a result of interactions would still obey a power law with possibly a different power $\alpha$. The power law expresses merely a certain distribution of baryon states and should not be completely obliterated by mass shifts. The plausibility of this conjecture is argued on the basis that the baryon-baryon interaction is not the dominant interaction even at very high densities, in contradistinction to the reasoning behind Zel'dovich's derivation of (3); the dominant interaction belongs indeed to the meson-baryon interaction which to a large extent is revealed by the baryon spectrum. (More explicit considerations of these arguments are given in Section 5). Hence, in the next section, we shall give a simple analytic study of the equation of state of dense baryon matter with an effective baryon mass spectrum given by (2) except that $\alpha$ is treated as a parameter, bearing in mind that even though the analysis resembles that of a free baryon gas, it has in fact a much broader basis of validity.

\section{The Equation of State}

Let us discuss now the statistical mechanics of a free baryon gas consisting of particles obeying a mass spectrum given by (2). The pressure $P$ and energy density $\mathscr{E}$ of such a gas are given by:

$$
\begin{aligned}
& P=\left(c_{2} / 3\right) \int \mathrm{d} m^{2} m^{2 \alpha} \int_{m}^{\infty} \frac{\left(\varepsilon^{2}-m^{2}\right)^{3 / 2} \mathrm{~d} \varepsilon}{e^{(\varepsilon-\mu) \beta}+1}, \\
& \mathscr{E}=c_{2} \int \mathrm{d} m^{2} m^{2 \alpha} \int_{m}^{\infty} \frac{\varepsilon^{2}\left(\varepsilon^{2}-m^{2}\right)^{1 / 2} \mathrm{~d} \varepsilon}{e^{(\varepsilon-\mu) \beta}+1},
\end{aligned}
$$

where $\mu$ is the chemical potential, $\beta=1 / k T$ and $c_{2}=c_{0}\left(2 \pi^{2} \hbar m_{N}{ }^{2 x+2}\right)^{-1}$. (Note that when there are a large variety of baryons present and occurring in different charge states, the role of leptons in the equation of state can be ignored.) 
Consider first the degenerate case where $\beta \rightarrow \infty$. Then,

$$
\begin{aligned}
P & =\left(c_{2} / 3\right) \int_{m_{0^{2}}}^{\mu^{2}} \mathrm{~d} m^{2} m^{2 \alpha} \int_{m}^{\mu}\left(\varepsilon^{2}-m^{2}\right)^{3 / 2} \mathrm{~d} \varepsilon, \\
\mathscr{E} & =c_{2} \int_{m_{0^{2}}}^{\mu^{2}} \mathrm{~d} m^{2} m^{2 \alpha} \int_{m}^{\mu} \varepsilon^{2}\left(\varepsilon^{2}-m^{2}\right)^{1 / 2} \mathrm{~d} \varepsilon \\
& =3 P+c_{2} \int_{m_{0^{2}}}^{\mu^{2}} \mathrm{~d} m^{2} m^{2(\alpha+1)} \int_{m}^{\mu}\left(\varepsilon^{2}-m^{2}\right)^{1 / 2} \mathrm{~d} \varepsilon,
\end{aligned}
$$

where the lower limit of the mass spectrum is denoted by $m_{0}^{2}$, which we may take to be $m_{0}^{2} \simeq 0.8 m_{\mathrm{N}}^{2}$. An integration by parts of the second term in the right-hand side of (7) gives:

$$
\mathscr{E}=3 P+2(\alpha+1) P+\Delta
$$

where

$$
\Delta=\left(2 c_{2} / 3\right) m_{0}^{2(\alpha+1)} \int_{m_{0}}^{\mu}\left(\varepsilon^{2}-m_{0}^{2}\right)^{3 / 2} \mathrm{~d} \varepsilon
$$

In this manner we have reduced the expression for $\mathscr{E}$ to one in terms of $P$ and $\Delta$. It is clear that the expression cannot be further reduced since $\Delta$ is only of the order of $P$, and $(\Delta / P)$ diminishes as $\mu$ increases at the rate $\left(m_{0} / \mu\right)^{2(\alpha+1)}$. The equation of state can now be written as

$$
(\mathscr{E}-\Delta)=(5+2 \alpha) P,
$$

which simplifies immediately to the form of (3) whenever $(\Delta / P)$ becomes negligible, viz.,

$$
\mathscr{E} \simeq(5+2 \alpha) P .
$$

Thus, the equation of state for cold dense matter is determined once $\alpha$ is known. In order for (11) to be useful, it is of course necessary that $\alpha$ should not vary to any extent as $\mu$ is varied. This, however, is expected to be the case since $\alpha$ appears as a power in the effective baryon mass spectrum and therefore should be comparatively stable.

To get some qualitative feeling for this analysis, we can adopt $\alpha=2$, which is the power derived for the known baryon spectrum. Assuming that the power law (2) holds up to a mass of $2.0 \mathrm{GeV}$ we shall consider the chemical potential $\mu$ also up to $2.0 \mathrm{GeV}$. Within the interval $1.5 \mathrm{GeV}<\mu<2.0 \mathrm{GeV}$, corresponding to matter densities $10^{17} \mathrm{~g} \mathrm{~cm}^{-3}<\varrho<2 \times 10^{18} \mathrm{~g} \mathrm{~cm}^{-3}$, the factor $(\Delta / P)$ is realized. The equation of state also compares well with the one using the actual mass of the baryons, as shown in Figure 4, testifying to the adequacy of the power law approximation to the baryon spectrum.

The analysis described above is restricted to the case of zero temperature in order to be precise. When the temperature is non-zero some of the mass states beyond the 
$2 \mathrm{GeV}$ limit will be excited and the mass spectrum may deviate from a simple power law there. The analysis is however not any more difficult when $\pi$ is finite. From inspection one can easily see that (10) is still correct provided that $\Delta$ and $P$ are modified by including the factor $[\exp (\varepsilon-\mu) \beta+1]^{-1}$ within the energy integral and that the upper limit of the mass integral no longer ends at $\mu^{2}$. Again, when $(\Delta / P)$ becomes negligible the equation of state reduces back to (11). There is however little need to consider the case of finite $\beta$ since the smallest energy scale in this problem is $m_{0}$ which corresponds to a temperature of $10^{13} \mathrm{~K}$.

Indeed, one can also consider a more general effective mass spectrum, such as:

$$
N=c_{0}\left(m^{2}\right)^{\alpha}\left(1+a_{1} m+a_{2} m^{2}+\cdots\right) .
$$

There is however very little to be gained by having a more complicated result at this time since we are only interested in estimating roughly at what densities the equation of state will take on the form given by (3).

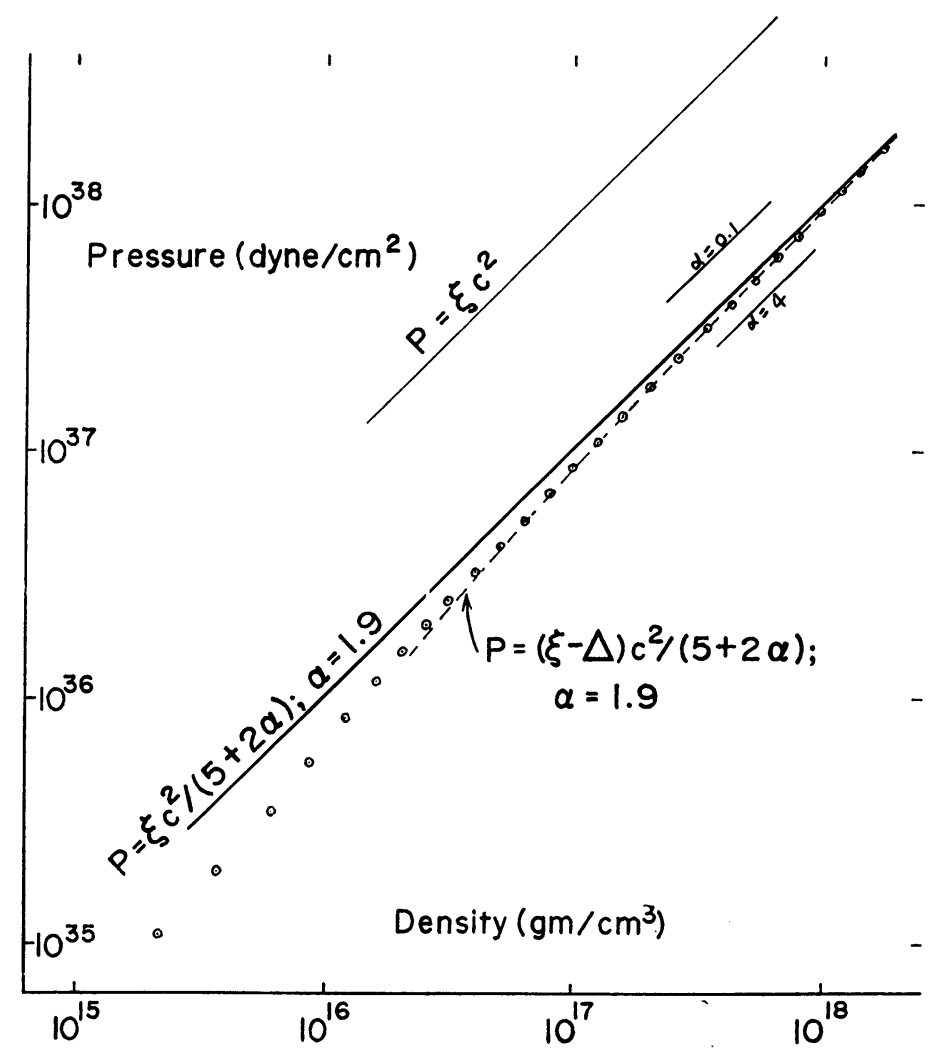

Fig. 4. The free baryon equation of state computed from Equation (10) using a simple power law approximation to the baryon spectrum is drawn in broken line, and the approximate expression given by Equation (11) is drawn in solid line. These are compared with that computed using the exact baryon spectrum. The latter is represented by circles. The equations of state are not sensitive to the index $\alpha$. 


\section{Discussion}

We try to point out in this article that the equation of state of matter at high densities takes on a particularly simple form given by (11) when supernuclear densities around $10^{17} \mathrm{~g} \mathrm{~cm}^{-3}$ are reached. We arrive at this result from dynamical considerations completely different from those employed before. Whereas it has been customary to assume that the interaction energy coming from inter-baryon repulsion would become dominant at high matter densities when baryons are squeezed very tightly together, we have avoided relying on this assumption completely since there are indications to the contrary (see below). Instead, we lay heavy emphasis on the meson-baryon interaction of which a great deal is revealed to us through the resonance spectrum of the baryons.

In nuclear physics, nuclear potentials are provided with a repulsive core. Such a strong short-range repulsive baryon-baryon interaction is deemed necessary to prevent nuclear collapse and finds its origin theoretically through the exchange of a neutral vector meson (like the $\omega$-meson). With such a repulsive core a baryon-baryon interaction will indeed play the dominant role for matter at high density. We can however infer its absence by extending the same theoretical basis for its presence, i.e., the mechanism of one-meson-exchange. The fact that spin-1 meson exchange produces repulsion and spin-2 meson exchange produces attraction between baryons can be easily seen through analogy with the Coulomb repulsion and gravitational attraction, which can be derived by the exchange of photon (spin-1) and graviton (spin-2) respectively, and in identifying the baryonic charge (i.e., the baryon number) with the electric charge and the gravitational mass. Recent studies in high energy particle physics indicate that the $\omega$-meson (spin-1) and the $f$-meson (spin-2), which are responsible for strong baryon-baryon forces, lie on 'exchange degenerate linear Regge trajectories,' which means the possible existence of (infinitely) many more even and odd spin mesons of increasingly high mass contributing alternatively to attractive and repulsive forces of comparable strengths between baryons. If this is the case, the net baryon-baryon interaction energy is not expected to be large at matter densities being considered. (At such densities the inter-baryon separation is of course considerably shorter than the ordinary nuclear separation and is well within the repulsive 'core' employed in nuclear physics.)

The dynamical situation is a great deal clearer with regard to meson-baryon interactions since the resonance spectrum provides us with useful information on the structure of the scattering amplitudes. The so-called 'narrow resonance models' in particle physics rely precisely on just such knowledge (see Sivers and Yellin, 1971 for a review). The implementation of the narrow resonance approximation for meson-baryon interaction in the framework of statistical mechanics is just the statement of (4) and (5) as made clear by the work of Dashen et al. (1969). Therefore, meson-baryon interactions can be handled reasonably adequately.

The remaining consideration centers upon mass shifts of the Sawyer type, which affect directly the treatment of the meson-baryon interaction discussed above. Since there exists at present no reliable means of estimating mass shifts for all baryon 
resonances, an exact determination of the equation of state of matter at supernuclear densities is impossible. We can nevertheless aim for a qualitative understanding of the equation of state at these densities. In line with the above discussion we postulate therefore an 'effective baryon mass spectrum' which includes the effects of mass shifts as well as baryon-baryon interactions. These effects are meant to cause minor distortions of the original baryon mass spectrum so that the power law approximation is retained. This then leads to our result given by (10) and (11).

Thus, we see that the equation of state of matter at supernuclear densities departs qualitatively from that of a free Fermi gas due to meson-baryon interaction which greatly undercuts the Fermi pressure in the system, and this is believed to occur at densities as low as $10^{17} \mathrm{~g} \mathrm{~cm}^{-3}$ using the known baryon mass spectrum as a guide.

We are grateful to Dr Philip Morrison who suggested to us the possibility of interpreting particle interactions in terms of a shifted mass within the free-particle formalism, a concept which we find useful here.

\section{Appendix A}

We list here the better known baryons which are being made use of in determining the baryon mass spectrum (see Figure 3). The data come from Söding et al. (1972) and their notations are being followed here. We use $m$ to denote the mass, $m_{N}$ the nucleon mass and $N$ the cumulative number of baryons below a certain mass. The statistics for $m$ below $2 \mathrm{GeV}$ is fairly accurate, but incomplete for higher masses.

\begin{tabular}{|c|c|c|c|c|c|c|}
\hline Name & Mass (MeV) & $\mathrm{m}^{2}\left(\mathrm{GeV}^{2}\right)$ & $\begin{array}{l}\text { Spin } \\
\text { Multiplets }\end{array}$ & $\begin{array}{l}\text { Isospin } \\
\text { Multiplets }\end{array}$ & $\begin{array}{l}\text { Total } \\
\text { Components }\end{array}$ & $N$ \\
\hline$N$ & 939 & 0.88 & 2 & 2 & 4 & 4 \\
\hline$\Lambda$ & 1115 & 1.24 & 2 & 1 & 2 & 6 \\
\hline$\Sigma$ & 1190 & 1.42 & 2 & 3 & 6 & 12 \\
\hline$\Delta$ & 1236 & 1.53 & 4 & 4 & 16 & 28 \\
\hline$\Xi$ & 1315 & 1.73 & 2 & 2 & 4 & 32 \\
\hline$\Sigma$ & 1385 & 1.92 & 4 & 3 & 12 & 44 \\
\hline$\Lambda$ & 1405 & 1.97 & 2 & 1 & 2 & 46 \\
\hline$N^{\prime}$ & 1470 & 2.16 & 2 & 2 & 4 & 50 \\
\hline$N^{\prime}$ & 1520 & 2.31 & 4 & 2 & 8 & 58 \\
\hline$\Lambda$ & 1520 & 2.31 & 4 & 1 & 4 & 62 \\
\hline$\Xi$ & 1530 & 2.34 & 4 & 2 & 8 & 70 \\
\hline$N^{\prime}$ & 1535 & 2.36 & 2 & 2 & 4 & 74 \\
\hline$\Delta$ & 1650 & 2.72 & 2 & 4 & 8 & 82 \\
\hline$\Delta$ & 1670 & 2.79 & 4 & 4 & 16 & 98 \\
\hline$N$ & 1670 & 2.79 & 6 & 2 & 12 & 110 \\
\hline$\Lambda^{\prime}$ & 1670 & 2.79 & 2 & 1 & 2 & 112 \\
\hline$\Sigma$ & 1670 & 2.79 & 4 & 3 & 12 & 124 \\
\hline $\boldsymbol{\Omega}$ & 1672 & 2.80 & 4 & 1 & 4 & 128 \\
\hline$N$ & 1688 & 2.85 & 6 & 2 & 12 & 140 \\
\hline$\Lambda^{\prime \prime}$ & 1690 & 2.86 & 4 & 1 & 4 & 144 \\
\hline
\end{tabular}


Appendix A (continued)

\begin{tabular}{|c|c|c|c|c|c|c|}
\hline Name & Mass(MeV) & $\mathrm{m}^{2}\left(\mathrm{GeV}^{2}\right)$ & $\begin{array}{l}\text { Spin } \\
\text { Multiplets }\end{array}$ & $\begin{array}{l}\text { Isospin } \\
\text { Multiplets }\end{array}$ & $\begin{array}{l}\text { Total } \\
\text { Components }\end{array}$ & $N$ \\
\hline$N^{\prime \prime}$ & 1700 & 2.89 & 2 & 2 & 4 & 148 \\
\hline$\Sigma$ & 1750 & 3.06 & 2 & 3 & 6 & 154 \\
\hline$\Sigma$ & 1765 & 3.12 & 6 & 3 & 18 & 172 \\
\hline$N^{\prime \prime}$ & 1780 & 3.17 & 2 & 2 & 4 & 176 \\
\hline$\Lambda$ & 1815 & 3.30 & 6 & 1 & 6 & 182 \\
\hline$\Xi$ & 1820 & 3.31 & 2 & 2 & 4 & 186 \\
\hline$\Lambda$ & 1830 & 3.35 & 6 & 1 & 6 & 192 \\
\hline$N$ & 1860 & 3.46 & 4 & 2 & 8 & 200 \\
\hline$\Delta$ & 1890 & 3.57 & 6 & 4 & 24 & 224 \\
\hline$\Delta$ & 1910 & 3.64 & 2 & 4 & 8 & 232 \\
\hline$\Sigma$ & 1915 & 3.66 & 6 & 3 & 18 & 250 \\
\hline$\Xi$ & 1940 & 3.76 & 2 & 2 & 4 & 254 \\
\hline$\Delta$ & 1950 & 3.80 & 8 & 4 & 32 & 286 \\
\hline$\Sigma$ & 2030 & 4.12 & 8 & 3 & 24 & 310 \\
\hline$\Lambda$ & 2100 & 4.41 & 8 & 1 & 8 & 318 \\
\hline$N$ & 2190 & 4.8 & 8 & 2 & 16 & 334 \\
\hline
\end{tabular}

\section{References}

Ambartsumyan, V. A. and Saakyan, G. S.: 1960, Astron. Zh. 37, 193. (English translation: 1960, Soviet Astron. - AJ 4, 187.)

Dashen, R., Ma, S. K., and Bernstein, H. J.: 1969, Phys. Rev. 187, 345.

Harrison, E. R.: 1965, Astrophys. J. 142, 1643.

Leung, Y. C. and Wang, C. G.: 1971, Astrophys. J. 170, 499.

Sawyer, R.: 1972a, Astrophys. J. 176, 205.

Sawyer, R.: 1972b, Phys. Rev. Letters 29, 382.

Scalapino, D. J.: 1972, Phys. Rev. Letters 29, 386.

Sivers, D. and Yellin, J.: 1971, Rev. Mod. Phys. 43, 125.

Söding, P., Bartels, J., Barbaro-Galtieri, A., Enstrom, J. E., Lasinski, T. A., Rittenberg, A., Rosenfeld, A. H., Trippe, T. G., Barash-Schmidt, N., Bricman, C., Chalorepka, V., and Roos, M.: 1972, Phys. Letters 39B, 1.

Zel'dovich, Ya: B.: 1961, Zh. Eksperim. i Teor. Fiz. 41, 1609 (English translation: 1962, Soviet Phys. JETP 14, 1143.)

\section{DISCUSSION}

Bethe: You have done something similar to Zel'dovich's work, but without strong repulsive interactions. Mind you that without strong repulsion, nuclei would collapse.

Wang: Most of the recent new nuclear potentials have more and more softer cores. I would be very surprised if anyone of them would give rise to a collapsed nuclei.

Bethe: In your calculation of the equation of state, instead of plotting $P$ and $E$, you should plot energy per baryon, which is more sensitive.

Wang: But $P$ and $E$ is what one uses to calculate the models of neutron stars. The main point of my talk is trying to show that at densities beyond $10^{17} \mathrm{~g} \mathrm{~cm}^{-3}$, the equation of state becomes insensitive to the detailed models of calculations.

Pandharipande: I differ with your statement that a soft repulsive core would give a much softer equation of state. My calculations with the Reid potential and with the BKR potential give very close results. You have that too in your graph. The problem is whether one should use a static potential.

Wang: My potential calculation has not been extended beyond the nuclear densities. I suppose 
that the relativistic effects would force all calculations to merge at the causality limit. But surely, at higher densities, the recoil momentum should be very important, therefore the non-static (non-local) contribution should be important. Mind you that Sawyer's criticism applies to the potential approach as well.

Pandharipande: Sawyer's self-energy is the three-body force term. Things like the relativistic corrections, the non-local recoil, the three-body forces, the three-body clustering etc. can be worked out in time. There is no reason to give up the potential approach.

Wang: What do we know about potentials among hadrons in general? Even the potentials among nucleons are difficult enough, and Sawyer's self-energy is not exactly the usual three-body force term. You have to worry about the self-consistency part of the Fermi energy, and you would get just as many coupled equations to solve. I can see some light to work out the nuclear physics from high energy physics, but not the other way around.

Pandharipandi: I would be most happy if your number and mine agree so we all do the right physics. I do not really care what approach one takes.

Wang: Come to think of it, we differ less than an order of magnitude, so there is really hope of convergence. 